\title{
Nanoscale inhomogeneities: A new path toward high Curie temperature ferromagnetism in diluted materials
}

\author{
Akash Chakraborty \\ Institut Néel, CNRS, Département MCBT, 25 avenue des Martyrs, \\ B.P. 166, 38042 Grenoble Cedex 09, France \\ Richard Bouzerar \\ Institut Néel, CNRS, Département MCBT, 25 avenue des Martyrs, \\ B.P. 166, 38042 Grenoble Cedex 09, France and \\ European Synchrotron Radiation Facility, \\ B.P. 220, F-38043 Grenoble Cedex, France \\ Stefan Kettemann \\ Division of Advanced Materials Science, \\ Pohang University of Science and Technology (POSTECH), \\ Pohang 790-784, South Korea and \\ School of Engineering and Science, Jacobs University Bremen, \\ Campus Ring 1, D-28759 Bremen, Germany \\ Georges Bouzerar \\ Institut Néel, CNRS, Département MCBT, 25 avenue des Martyrs, \\ B.P. 166, 38042 Grenoble Cedex 09, France and \\ School of Engineering and Science, Jacobs University Bremen, \\ Campus Ring 1, D-28759 Bremen, Germany
}

(Dated: April 26, 2022) 


\begin{abstract}
Room temperature ferromagnetism has been one of the most sought after topics in today's emerging field of spintronics. It is strongly believed that defect- and inhomogeneity- free sample growth should be the optimal route for achieving room-temperature ferromagnetism and huge efforts are made in order to grow samples as "clean" as possible. However, until now, in the dilute regime it has been difficult to obtain Curie temperatures larger than that measured in well annealed samples of $(\mathrm{Ga}, \mathrm{Mn}) \mathrm{As}$ ( $190 \mathrm{~K}$ for $12 \%$ doping). In the present work, we propose an innovative path to room temperature ferromagnetism in diluted magnetic semiconductors. We theoretically show that even a very small concentration of nanoscale inhomogeneities can lead to a tremendous boost of the critical temperatures: up to a $1600 \%$ increase compared to the homogeneous case. In addition to a very detailed analysis, we also give a plausible explanation for the wide variation of the critical temperatures observed in $(\mathrm{Ga}, \mathrm{Mn}) \mathrm{N}$ and provide a better understanding of the likely origin of very high Curie temperatures measured occasionally in some cases. The colossal increase of the ordering temperatures by nanoscale cluster inclusions should open up a new direction toward the synthesis of materials relevant for spintronic functionalities.
\end{abstract}

PACS numbers: 75.50.Pp, 75.30.Kz, 75.40.-s 


\section{INTRODUCTION}

The hope of attaining ferromagnetic order at room temperature and above, has spurred a huge interest in the field of diluted magnetic semiconductors (DMSs) ${ }^{1-3}$ and diluted magnetic oxides (DMOs) ${ }^{4}$. Extensive experimental as well as theoretical efforts have been made to predict high Curie temperatures $\left(\mathrm{T}_{C}\right)$ in these materials. Among the various materials widely studied, one of particular interest is (Ga,Mn)N, a wide-band-gap DMS. Different experimental results have reported $\mathrm{T}_{C}$ 's varying as widely as 10 to $940 \mathrm{~K}^{17-11}$ with a typical $\mathrm{Mn}$ content between 7 and $9 \%$. However, recent theoretical studies, based on model calculations, have predicted a $\mathrm{T}_{C}$ of $30 \mathrm{~K}$ in homogeneously diluted and uncompensated $\mathrm{Ga}_{1-x} \mathrm{Mn}_{x} \mathrm{~N}$ for $x=0.06^{12}$, which is in good agreement with results obtained from $a b$ initio based studies 13 combined with the self-consistent local random-phase approximation (SC-LRPA) method. On the other hand, using the same ab initio couplings, the Monte Carlo studies lead to $\mathrm{T}_{C}$ of $35 \mathrm{~K}$ for $x=0.06^{\frac{1415}{115}}$ in $\mathrm{Ga}_{1-x} \mathrm{Mn}_{x} \mathrm{~N}$. These theoretical calculations predict the highest reachable $\mathrm{T}_{C}$ in homogeneously diluted $\mathrm{Ga}_{1-x} \mathrm{Mn}_{x} \mathrm{~N}$. Then how can we explain the very high Curie temperatures observed by some experimental groups? We will provide an answer in the following.

From these observations crucial questions arise. How do we explain the huge fluctuations of the critical temperatures in these materials? Is there a systematic way to boost the critical temperatures beyond that expected in the homogeneous compounds (inhomogeneity free)? After observation of ferromagnetic order in Mn-doped Germanium $\left(\mathrm{T}_{C}=116\right.$ $\mathrm{K}$ for $x=0.035)^{16}$, several experimental studies reported quite high critical temperatures in $(\mathrm{Ge}, \mathrm{Mn})$ films ${ }^{17}$.20 . However, the underlying reasons were not really clear. In Ref. ${ }^{21}$ scanning photoelectron microscopy measurements revealed stripe-shaped Mn rich microstructures which were believed to be the origin of ferromagnetism in $\mathrm{Ge}_{1-x} \mathrm{Mn}_{x}$. More recent experimental studies have revealed self-organized $\mathrm{Mn}$ rich nanocolumns formation in $\mathrm{Ge}_{1-x} \mathrm{Mn}_{x}$, which gave rise to a very high $\mathrm{T}_{C}(\geq 400 \mathrm{~K})^{22}$ for $x=0.06$. Magnetotransport measurements, in this case, have also shown a large anomalous Hall effect up to room temperature. The spinodal decomposition (alternating regions of low and high concentration of magnetic impurities) was suggested to be the reason for the high temperature ferromagnetism in this case. Similar nanometer-sized clusters, with increased Mn content compared to the surrounding matrix, were also detected by transmission electron microscopy (TEM) analysis 
in $\mathrm{Ge}_{0.95} \mathrm{Mn}_{0.05} \sqrt{23}$. In recent experimental studies on $(\mathrm{Zn}, \mathrm{Co}) \mathrm{O}^{24}$, the authors claimed the existence of two types of nanosized ferromagnetic Co clusters. The first were spherical with diameters of about $5 \mathrm{~nm}$ leading to critical temperatures of $\sim 100 \mathrm{~K}$ and the others were columnar about $4 \mathrm{~nm}$ wide, with a maximum height of $60 \mathrm{~nm}$, leading to significantly larger critical temperatures of $\sim 300 \mathrm{~K}$. These results were confirmed by high-resolution transmission electron microscopy (HRTEM). Hence this kind of anisotropic nanoscale inhomogeneity can lead to interesting magnetic and transport properties. Inspite of the existence of several experimental studies, the effect of impurity clustering on magnetism in DMSs and DMOs has been weakly studied on the theoretical front. Ab initio based studies for these type of inhomogeneous disordered systems are difficult due to the large size of supercells required and no standard methods have been proposed as yet. In Ref. ${ }^{25}$ the authors have simulated the spinodal decomposition in DMS by using Monte Carlo methods and they have predicted an above-room-temperature $\mathrm{T}_{C}$ for the spinodal phase in $(\mathrm{Ga}, \mathrm{Mn}) \mathrm{As}$ and $(\mathrm{Ga}, \mathrm{Mn}) \mathrm{N}$, calculated from the "standard" random-phase approximation (RPA). Here "standard" means that the crucial self consistency was not implemented in the RPA calculations. However these high $\mathrm{T}_{C}$ s were found for samples containing a relatively high concentration of Mn, above $20 \%$, far from the dilute regime. On the other hand in the dilute case, for approximately $5 \%$ of Mn, the authors have found a suppression of the critical temperatures in the presence of spinodal decomposition phases. Note also that the calculations were limited to small system sizes compared to the typical size of the inhomogeneities and the average was done over few configurations only (typically 10). In Ref. $\stackrel{26}{t}$ the authors have presented density-functional theory (DFT) based calculations of N-doped Mn clusters, and have given a hypothesis that a high Curie temperature detected in some of the GaMnN samples is a result of the formation of small Mn clusters carrying giant magnetic moments. The large variation in Curie temperatures could be attributed to the formation of $\mathrm{N}$ induced $\mathrm{Mn}$ clusters of different sizes in samples grown under different conditions. Their analysis suggests the importance of the growth mechanism in these kind of materials. Similar density-functional calculations on the effect of microscopic Mn clustering on the Curie temperatures of $(\mathrm{Ga}, \mathrm{Mn}) \mathrm{N}$ were also reported in Ref.27. However the $\mathrm{T}_{C}$ 's were calculated from the mean-field approximation, which is already known to overestimate the Curie temperatures in homogeneously diluted semiconductors.

In this article, we present a generalized and comprehensive study of the effect of nanoscale 

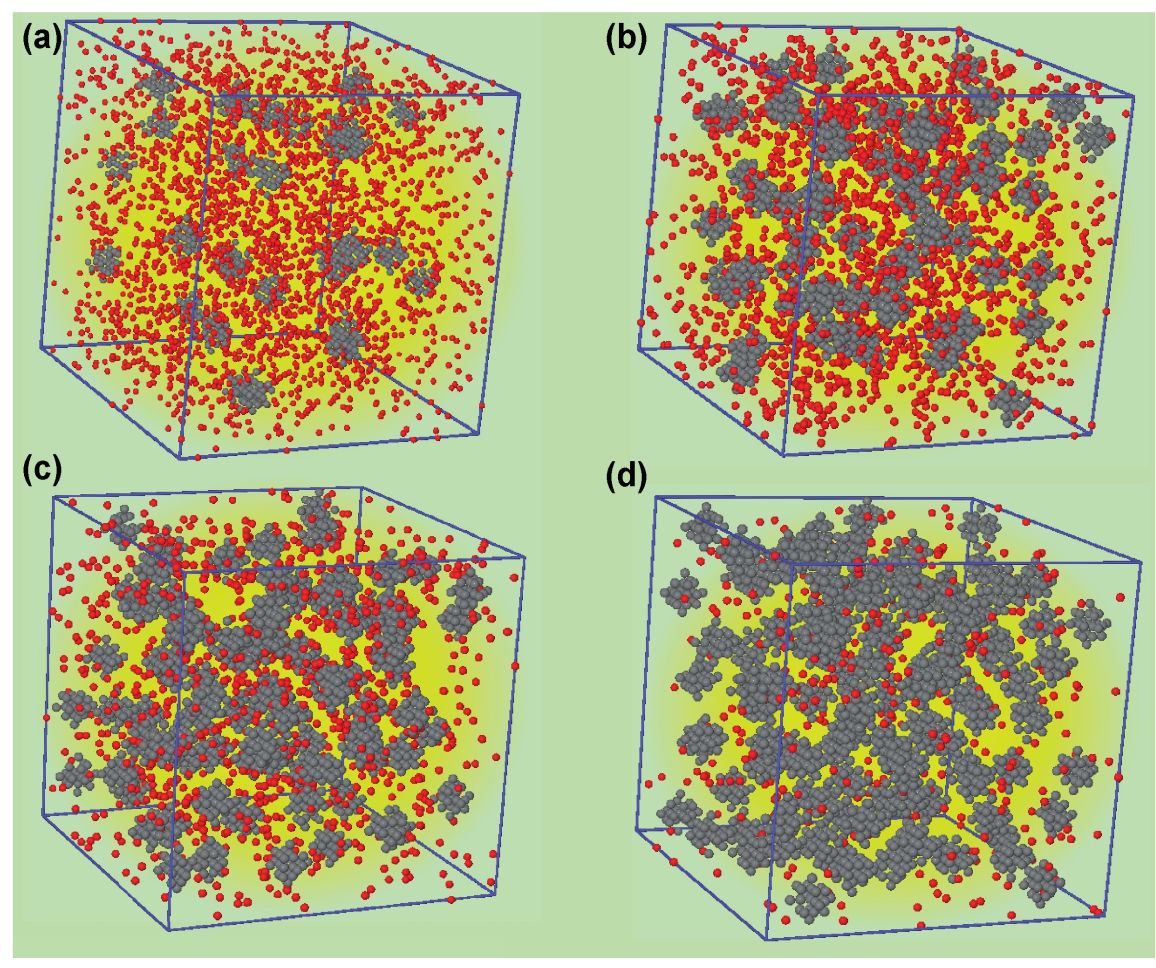

FIG. 1: (Color online)Snapshots corresponding to four different concentrations of nanospheres $x_{n s}$ (a) 0.02 , (b) 0.04, (c) 0.06 and (d) 0.08. The grey (red) atoms denote the impurities inside (outside) the nanospheres. Here $\mathrm{L}=36, \mathrm{r}_{0}=2 a$ ( $a$ is the lattice spacing) and $x_{i n}=0.8$.

inhomogeneities on the Curie temperatures in diluted magnetic systems. The calculations are performed on very large systems (finite size analysis is provided) and a systematic sampling is done over several hundreds of disorder configurations. In contrast to previous studies, we report giant effects on the $\mathrm{T}_{C}$ in dilute materials. In some particular cases the $\mathrm{T}_{C}$ can be enhanced by up to $1600 \%$ compared to that of the homogeneously diluted system. There are several factors that lead to these effects, such as the concentration of inhomogeneities in the system, the size of the inhomogeneities, the concentration of magnetic impurities inside the inhomogeneities and also the range of the exchange interactions between the impurities. In the following we shall see how these physical parameters play an important role and affect the critical temperatures. 


\section{MODEL AND METHOD}

For simplicity we have assumed here a simple cubic crystalline structure and the conclusions that will be drawn will be general. The sizes vary from $\mathrm{L}=32$ to $\mathrm{L}=44$. The inhomogeneities considered here are of spherical shape of radii $\mathrm{r}_{0}$. For the sake of clarity and to avoid additional parameters, in our calculations the total concentration of impurities in the whole system is fixed to $x=0.07$. In the following we denote the concentration of nanospheres by $x_{n s}=\mathrm{N}_{S} / \mathrm{N}$, where $\mathrm{N}_{S}$ is the total number of sites included in all the nanospheres and $\mathrm{N}=\mathrm{L}^{3}$ is the total number of sites. The concentration of impurities inside each nanosphere is defined by $x_{i n}$. We denote the total number of impurities and the number of impurities inside the nanospheres by $\mathrm{N}_{i m p}^{t o t}$ and $\mathrm{N}_{i m p}^{i n}\left(=x_{i n} \mathrm{~N}_{S}\right)$ respectively. We choose the nanospheres in such a manner so as to restrict their overlap with each other.

In Fig. 1 four typical random configurations corresponding to four different concentration of nanospheres $x_{n s}(0.02,0.04,0.06$ and 0.08$)$ are depicted. As we increase the number of nanospheres in the system $x_{n s}$ increases and consequently the concentration outside decreases, since the total concentration $(x)$ is fixed. Now to evaluate the $\mathrm{T}_{C}$, the effective diluted Heisenberg Hamiltonian $\mathrm{H}_{\text {Heis }}=-\sum_{i, j} \mathrm{~J}_{i j} \mathbf{S}_{i} \cdot \mathbf{S}_{j}$, is treated within the SC-LRPA theory. The self-consistent local RPA is a semi-analytical approach based on finite temperature Green's functions. It is essentially an extension of the standard RPA to the case of disordered systems. Here the thermal fluctuations are treated within the RPA and the disorder is treated exactly without any approximations. The Curie temperature of a system containing $N_{i m p}$ localized spins is obtained from the expression

$$
k_{B} T_{C}=\frac{2}{3} S(S+1) \frac{1}{N_{i m p}} \sum_{i} \frac{1}{F_{i}}
$$

where

$$
F_{i}=-\frac{1}{2 \pi \lambda_{i}} \int_{-\infty}^{\infty} \frac{\Im G_{i i}(E)}{E} d E
$$

We define the retarded Green's function as $G_{i j}(\omega)=\int_{-\infty}^{\infty} G_{i j}(t) e^{i \omega t} d t=\left\langle\left\langle S_{i}^{+} ; S_{j}^{-}\right\rangle\right\rangle$. The set of parameters $\lambda_{i}=\lim _{T \rightarrow T_{C}}\left\langle S_{i}^{z}\right\rangle / m$, where $m$ is the average magnetization, are calculated self-consistently (more details can be found in Ref ${ }^{1113}$ ). The $\mathrm{T}_{C}$ is calculated for each random configuration and then averaged over a few hundred configurations of disorder. The accuracy and reliability of the SC-LRPA to treat disorder and/or dilution has been demonstrated 
TABLE I: The ratio, $\left\langle\mathrm{R}^{\text {hom }}\right\rangle$, of the homogeneous Curie temperatures for different $x$ to that of $x=0.07$, for $\lambda=a$ and $a / 2$

\begin{tabular}{lcc}
\hline \hline$x$ & $\left\langle\mathrm{R}^{\text {hom }}\right\rangle(\lambda=a)$ & $\left\langle\mathrm{R}^{\text {hom }}\right\rangle(\lambda=a / 2)$ \\
\hline 0.8 & 9.7 & 22 \\
0.7 & 8.9 & 20 \\
0.6 & 7.9 & 17 \\
0.4 & 5.2 & 11 \\
\hline \hline
\end{tabular}

several times in the past $\mathrm{t}^{1112 \mid 28}$.

The exchange couplings in a DMS, as found from ab initio based calculations, are rel-

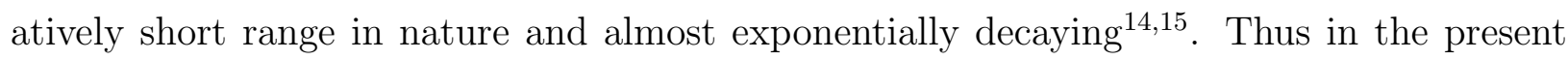
study we have assumed generalized couplings of the form $J_{i j}=J_{0} \exp (-|\mathbf{r}| / \lambda)$, where $\mathbf{r}=\mathbf{r}_{i}-\mathbf{r}_{j}$ and $\lambda$ is the damping parameter. In (Ga,Mn)As, for about $5 \% \mathrm{Mn}$ a fit of the $a b$ initio magnetic couplings provides a value of $\lambda$ of the order of a/2. Note that in the case of (Ga,Mn)N the ab initio couplings are of even shorter range. Thus we focus here on two particular cases, $\lambda=a$ and $\lambda=a / 2$, where $a$ is the lattice spacing. Although these length scales are comparable, in the presence of inhomogeneities the effects on the critical temperatures will be very drastic. In order to measure directly the effects of nanoscale inhomogeneities, the averaged Curie temperatures $\left\langle T_{C}^{i n h}\right\rangle$ are scaled with respect to the averaged Curie temperatures of the homogeneously diluted system $\left\langle T_{C}^{h o m}\right\rangle$ for $x=0.07$, their ratio is denoted by $\left\langle\mathrm{R}_{C}\right\rangle$. The averaged Curie temperatures $\left\langle T_{C}^{\text {hom }}\right\rangle$ for the homogeneous systems are found to be $0.9 \mathrm{~J}_{0}$ and $0.05 \mathrm{~J}_{0}$, for $\lambda=a$ and $a / 2$ respectively, for $x=0.07$.

In Table (I) we provide the averaged Curie temperatures for $80 \%, 70 \%, 60 \%$ and $40 \%$ homogeneously distributed magnetic impurities scaled with respect to that of the $7 \%$ homogeneous case, for $\lambda=a$ and $a / 2$. The ratio is denoted by $\left\langle\mathrm{R}^{\text {hom }}\right\rangle$. These values will be relevant in the discussions to follow, where we consider these types of concentrations inside the nanospheres. 

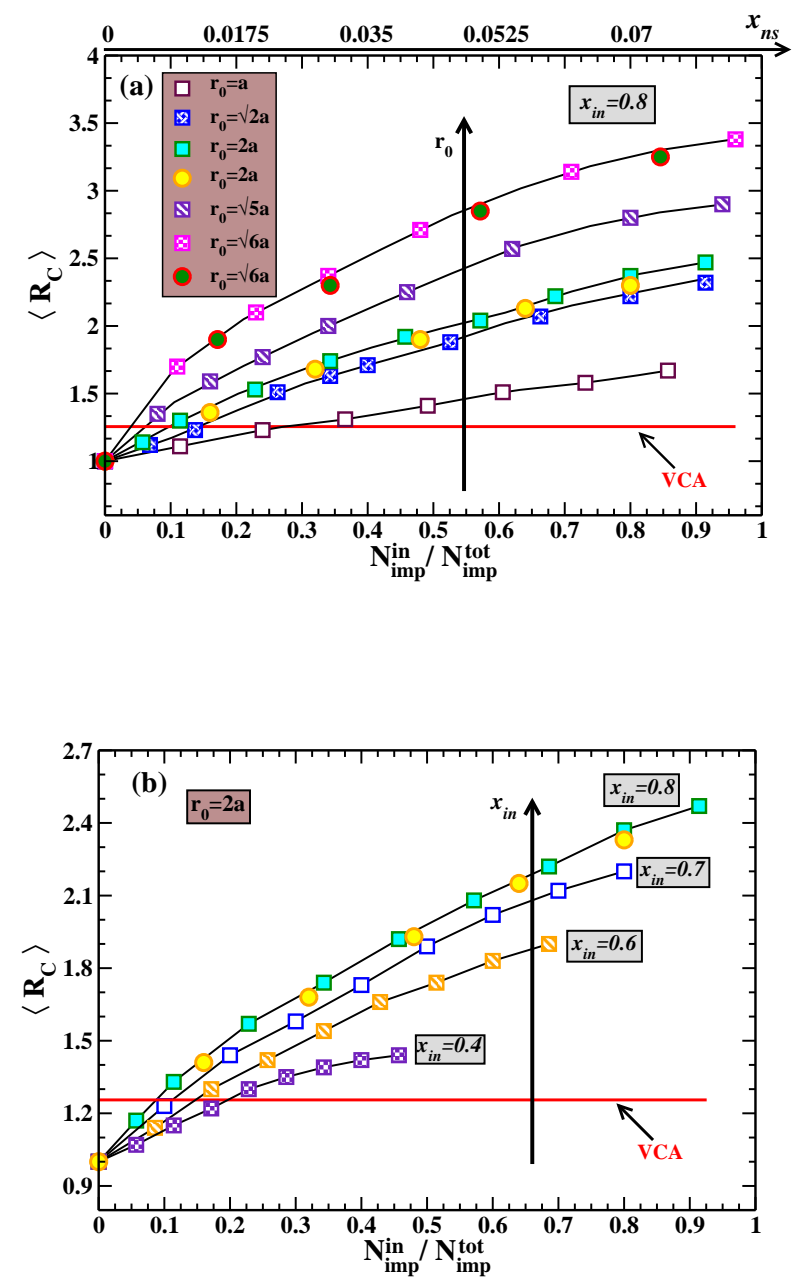

FIG. 2: (Color online) $\left\langle\mathrm{R}_{C}\right\rangle=\frac{\left\langle T_{C}^{i n h}\right\rangle}{\left\langle T_{C}^{h o m}\right\rangle}$ as a function of $\mathrm{N}_{i m p}^{i n} / \mathrm{N}_{i m p}^{t o t}=\frac{x_{i n}}{x} x_{n s}$ for $\lambda=a$. (a) Results for a fixed concentration inside the nanospheres $\left(x_{i n}=0.8\right)$ and different radii $\mathrm{r}_{0}$. The upper $x$ axis represents the values of $x_{n s}$ corresponding to $x_{i n}=0.8$. The long black arrow indicates the direction of increasing $\mathrm{r}_{0}$. (b) Results for a fixed radius $\left(\mathrm{r}_{0}=2 a\right)$ and different concentration inside the nanospheres. The long black arrow indicates the direction of increasing $x_{i n}$. The solid red line indicates the $\mathrm{T}_{C}^{V C A}$ scaled with respect to $\left\langle T_{C}^{h o m}\right\rangle$. In the figures, squares correspond to $\mathrm{L}=32$ and circles to $\mathrm{L}=36$.

\section{RESULTS AND DISCUSSION}

Figure 2(a) shows $\left\langle\mathrm{R}_{C}\right\rangle$ as a function of $\mathrm{N}_{i m p}^{i n} / \mathrm{N}_{i m p}^{t o t}$ corresponding to the case of $\lambda=a$. The concentration inside the nanospheres is fixed at $x_{i n}=0.8$ and the $\mathrm{T}_{C}$ is calculated for spheres of different radii. For this concentration inside $x_{i n}$, each nanosphere contains $5,15,26,45$, 
and 64 impurities for $\mathrm{r}_{0}=a, \sqrt{2} a, 2 a, \sqrt{5} a$, and $\sqrt{6} a$ respectively. $\mathrm{N}_{i m p}^{i n} / \mathrm{N}_{i m p}^{\text {tot }}=0$ corresponds to the homogeneously diluted case (absence of inhomogeneities). We observe a clear increase in the critical temperatures with increasing fraction of impurities inside the nanospheres as well as with the nanospheres' size. For about $80 \%$ of the total impurities inside the nanospheres, $\mathrm{T}_{C}$ is enhanced by up to $150 \%$ for the smallest nanospheres with $\mathrm{r}_{0}=a$, and by almost $350 \%$ for the ones of radius $\mathrm{r}_{0}=\sqrt{6} a$, which is rather significant. This increase for $\mathrm{r}_{0}=\sqrt{6} a$ is more than one-third of that found for the $80 \%$ homogeneously distributed case (Table II). Thus the clustering of magnetic impurities does lead to a considerable increase of the critical temperatures due to the strong interactions within the nanospheres. The other important point to take note of is the $\mathrm{T}_{C}$ obtained from the mean field virtual crystal approximation (VCA), $\mathrm{T}_{C}^{V C A}=\frac{2}{3} x \sum_{i} \mathrm{n}_{i} \mathrm{~J}_{i}$, where $\mathrm{n}_{i}$ is the number of atoms in the $i$-th shell. It is well known that the VCA overestimates the true critical temperatures, often very strongly. However, the present results show that in the presence of inhomogeneities the VCA value can no longer serve as an upper bound. Indeed, as can be seen here, for a relatively small concentration of nanospheres $\left(x_{n s} \sim 0.2\right)$ the VCA value is already exceeded and for higher density of nanospheres the VCA actually strongly underestimates the critical temperatures in these systems.

Let us now focus on the case where the nanospheres are of fixed radius $\left(\mathrm{r}_{0}=2 a\right)$ and the concentration inside the nanospheres vary $\left(\mathrm{Fig} .2(\mathrm{~b}) \cdot\left\langle\mathrm{R}_{C}\right\rangle\right.$ is plotted as a function of $\mathrm{N}_{i m p}^{i n} / \mathrm{N}_{i m p}^{t o t}$ for different $x_{i n}$. The curves show an overall monotonous increase with increasing concentration of nanospheres. However, the enhancement of the critical temperatures is also controlled by the concentration of impurities inside the nanospheres. Decreasing the concentration inside the nanospheres effectively means reducing the number of impurities inside a cluster of the same size, and thus reducing inter nanosphere interactions. This could explain the relatively small increase in the $\mathrm{T}_{C}$ values with decreasing $x_{i n}$. However, as will be seen in the following, the variation of the critical temperatures is more complex than this simple picture. Thus we find that not only the relative number of impurities inside the nanospheres but also the concentration inside the nanospheres have a drastic effect on the critical temperatures in these systems.

Now we move to the case of the shorter-ranged couplings, $\lambda=a / 2$, which will appear even more interesting and which lead to unexpected effects. Figure $3(\mathrm{a})$ shows the $\left\langle\mathrm{R}_{C}\right\rangle$ as a function of $\mathrm{N}_{i m p}^{i n} / \mathrm{N}_{i m p}^{t o t}$ for a fixed $x_{i n}=0.8 . \mathrm{T}_{C}$ is calculated for nanospheres of different radii 

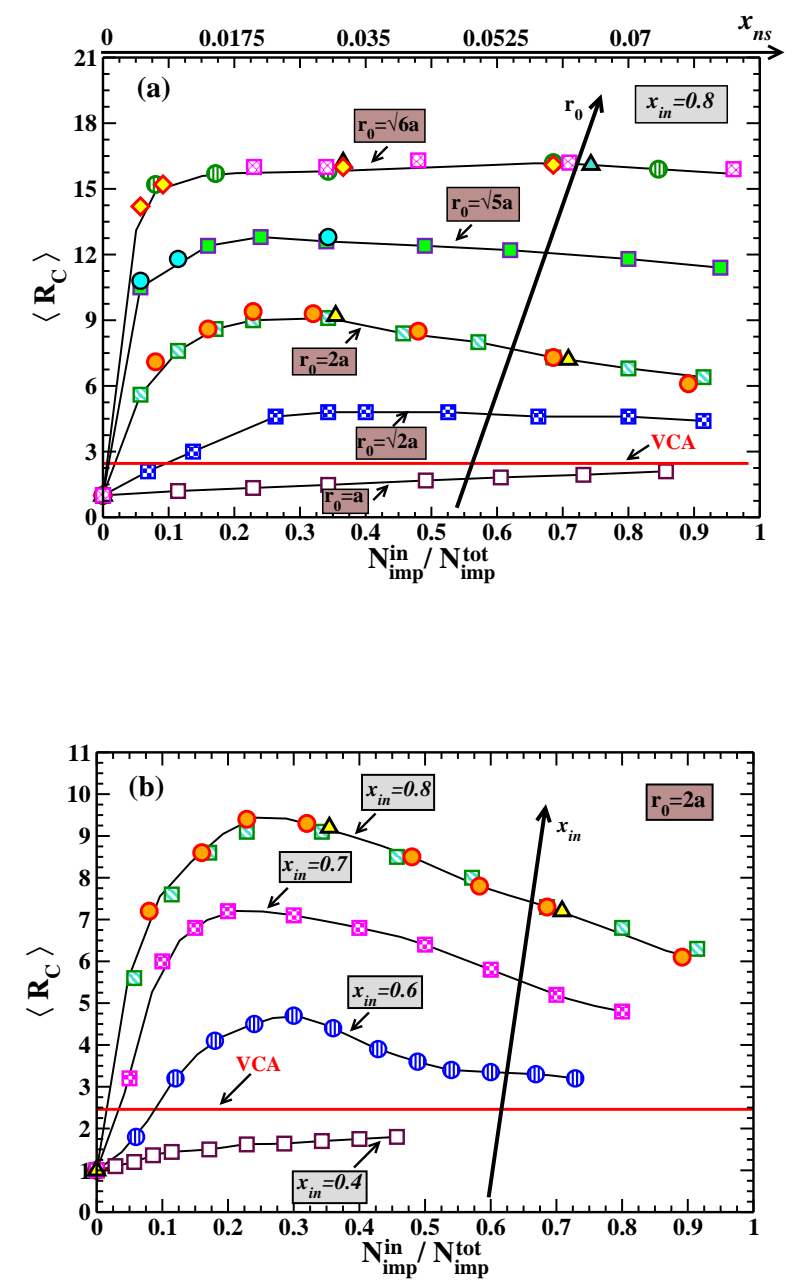

FIG. 3: (Color online) $\left\langle\mathrm{R}_{C}\right\rangle=\frac{\left\langle T_{C}^{i n h}\right\rangle}{\left\langle T_{C}^{h o m}\right\rangle}$ as a function of $\mathrm{N}_{i m p}^{i n} / \mathrm{N}_{i m p}^{t o t}=\frac{x_{i n}}{x} x_{n s}$ corresponding to $\lambda=a / 2$. (a) Results for a fixed concentration inside the nanospheres $\left(x_{i n}=0.8\right)$ and different radii $\mathrm{r}_{0}$. The upper $x$ axis represents the values of $x_{n s}$ corresponding to $x_{i n}=0.8$. The long black arrow indicates the direction of increasing $\mathrm{r}_{0}$. (b)Results for a fixed radius $\left(\mathrm{r}_{0}=2 a\right)$ and different concentration inside the nanospheres. The long black arrow indicates the direction of increasing $x_{i n}$. The solid red line indicates the $\mathrm{T}_{C}^{V C A}$ scaled with respect to $\left\langle T_{C}^{h o m}\right\rangle$. In the figures, squares correspond to $\mathrm{L}=32$, circles to $\mathrm{L}=36$, triangles to $\mathrm{L}=40$, and diamonds to $\mathrm{L}=44$.

$\left(\mathrm{r}_{0}=a, \sqrt{2} a, 2 a, \sqrt{5} a\right.$ and $\left.\sqrt{6} a\right)$. We have considered system sizes varying from $\mathrm{L}=32$ to $\mathrm{L}=44$ to check for the finite-size effects. The $\mathrm{L}=44$ systems typically contain $\sim 6000$ impurities. In contrast to the case of $\lambda=a$ discussed above, the variation of $\mathrm{T}_{C}$ with $\mathrm{N}_{i m p}^{\text {in }} / \mathrm{N}_{i m p}^{\text {tot }}$ is not monotonous anymore. Here we see a colossal effect of the size of the nanospheres on the 
$\mathrm{T}_{C}$. For the smallest nanospheres $\left(\mathrm{r}_{0}=a\right)$ there is hardly any noticeable effect, with the critical temperatures remaining close to that of the homogeneous case. Now as we increase the radius of the nanospheres for a given concentration of nanospheres, there is a sharp and strong increase in the $\mathrm{T}_{C}$ values. As can be seen, even for a reasonably small concentration of nanospheres $\left(x_{n s} \sim 0.2\right)$ we obtain a remarkable jump of almost $900 \%$ for $\mathrm{r}_{0}=2 a$ and even $1600 \%$ for $\mathrm{r}_{0}=\sqrt{6} a$, compared to that of the homogeneous case. This gigantic increase in the presence of nanospheres with $\mathrm{r}_{0}=\sqrt{6} a$ is more than $70 \%$ when compared to the $\mathrm{T}_{C}$ of the $80 \%$ homogeneous case (Table I), which is rather extraordinary. This implies that in materials such as $(\mathrm{Ga}, \mathrm{Mn}) \mathrm{N}$, where the exchange interactions are really short ranged, it would be possible to reach $\mathrm{T}_{C} \geq 500 \mathrm{~K}$ (as $\mathrm{T}_{C}$ for homogeneously diluted $\mathrm{Ga}_{1-x} \mathrm{Mn}_{x} \mathrm{~N}$ is $40 \mathrm{~K}$ for $x=0.07-12+14)$ by inducting nanoscale inhomogeneities. The presence of such nanoclusters may also explain the very high $\mathrm{T}_{C}$ observed in $\mathrm{Ga}_{1-x} \mathrm{Mn}_{x} \mathrm{~N}$ by some experimental groups ${ }^{111}$. It should be of great interest to analyse experimentally the effect of such nanoclusters on the critical temperatures in these kind of materials. Here again the mean field VCA is found to strongly underestimate the $\mathrm{T}_{C}$ for most cases. This is expected since the mean field VCA treatment is unable to capture all the relevant physical effects in both homogeneously disordered as well as inhomogeneous systems. Thus it becomes clear that in systems with relatively short- ranged couplings the size of the inhomogeneities plays a very important role in controlling the critical temperatures. The nonmonotonous behavior observed here implies that several physical parameters are in competition (length scales and relevant couplings). Thus, we cannot explain this variation by assuming the inhomogeneities to behave as "superspins" only.

In Fig. 3(b) we consider the case of nanospheres of fixed radii $\mathrm{r}_{0}=2 a$, which is particularly interesting. $\left\langle\mathrm{R}_{C}\right\rangle$ is shown as a function of $\mathrm{N}_{i m p}^{i n} / \mathrm{N}_{i m p}^{\text {tot }}$ for different $x_{i n}(0.8,0.7,0.6$ and 0.4$)$. For a fixed $x_{i n}$, we observe a gradual increase in the critical temperatures with increasing concentration of nanospheres, and then it decreases as $x_{n s}$ increases further. In contrast to the case of $\lambda=a$, there is a clear maximum in the $\mathrm{T}_{C}$ around $\mathrm{N}_{i m p}^{i n} / \mathrm{N}_{i m p}^{\text {tot }} \sim 0.2$ for $x_{i n}=80 \%$ and $70 \%$. For this value of $\mathrm{N}_{i m p}^{i n} / \mathrm{N}_{i m p}^{t o t}$, as we increase the concentration inside the nanospheres we observe a huge jump in the critical temperatures, from a small increase for $x_{i n}=40 \%$ to almost $900 \%$ for $x_{i n}=80 \%$, compared to that of the homogeneous case. It should be noted that for $\mathrm{N}_{i m p}^{i n} / \mathrm{N}_{i m p}^{t o t}=0.9$ and $x_{i n}=80 \%$ the increase is reduced to about $600 \%$, which is still considerably large. However for $x_{i n}=40 \%$ we hardly obtain any significant increase 
compared to that of the homogeneous case. Hence in this case the concentration inside the nanospheres is found to have a crucial effect on determining the critical temperatures of the system. A careful statistical analysis reveals that the case of $\mathrm{r}_{0}=2 a$ for $\lambda=a / 2$ is particularly intriguing. As will be seen, the analysis of the $\mathrm{T}_{C}$ distributions exhibits interesting features. In the following we provide a more detailed study for this particular case and try to analyse the reasons for the origin of this kind of behavior.

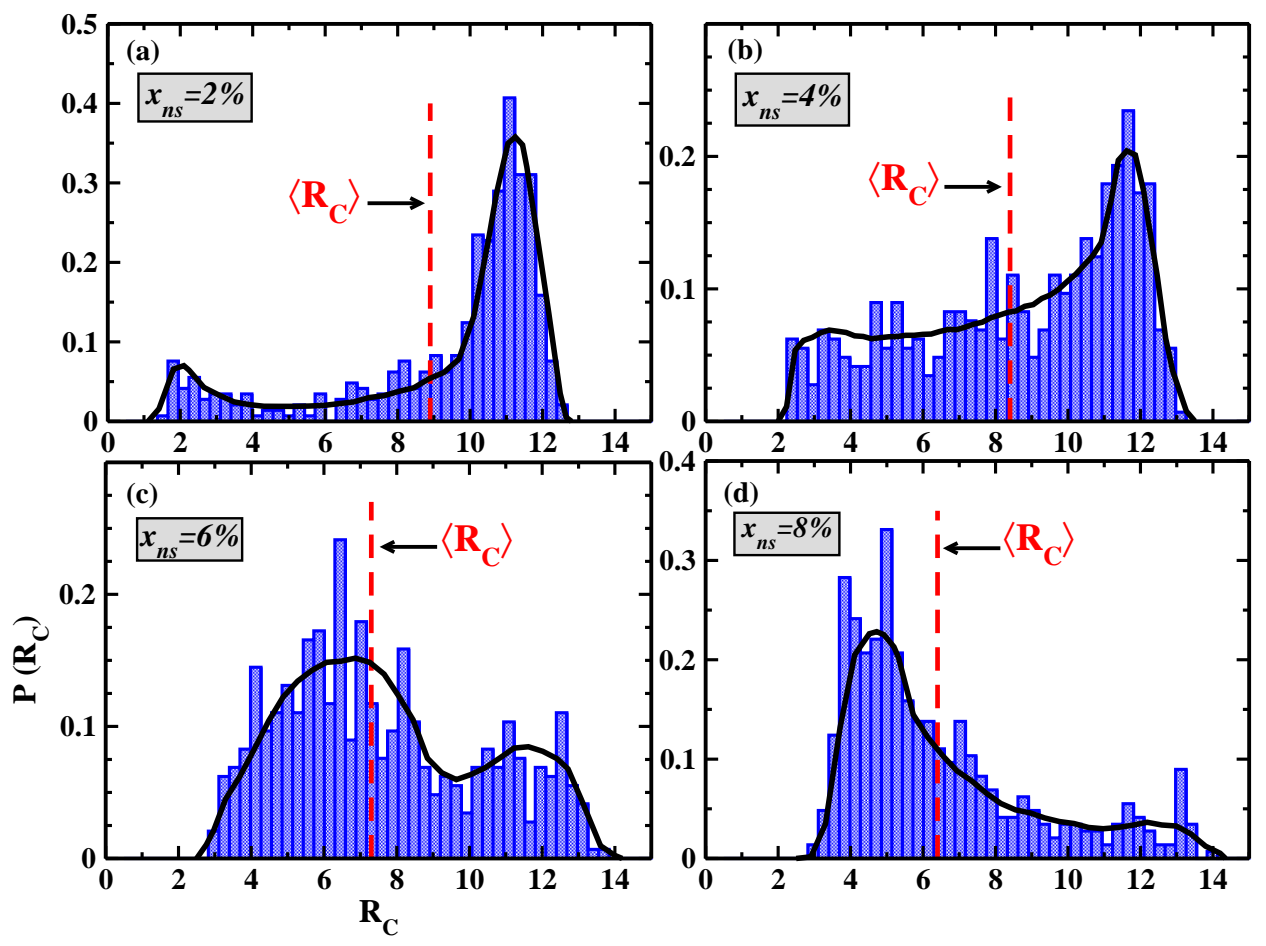

FIG. 4: (Color online)Normalized $\mathrm{R}_{C}$ distributions for four different $x_{n s}$ : (a) 0.02, (b) 0.04, (c) 0.06 , and (d) 0.08 corresponding to $\lambda=a / 2$. Here $\mathrm{r}_{0}=2 a, x_{i n}=0.8$ and $\mathrm{L}=32$. The red dashed lines indicate the $\left\langle\mathrm{R}_{C}\right\rangle$ values, which we have shown in Fig $3(\mathrm{~b})$, The solid black lines are a guide to the eye.

In Fig. 4 we show the normalized $\mathrm{R}_{C}$ distributions corresponding to the case of $\mathrm{r}_{0}=2 a$, $x_{i n}=0.8$ and $\lambda=a / 2$. The distributions are obtained using a sampling over a few hundred configurations of disorder ( 600$)$. As can be seen from the figure, we obtain very interesting wide distributions for the different concentrations of nanospheres $\left(x_{n s}=0.02,0.04,0.06\right.$ and $0.08)$. For $x_{n s}=0.02$ we observe a kind of bimodal distribution: one peak with a large weight at high $\mathrm{T}_{C}\left(\mathrm{~T}_{C}^{\text {high }} \sim 11\left\langle T_{C}^{\text {hom }}\right\rangle\right)$ values and another one at lower $\mathrm{T}_{C}\left(\mathrm{~T}_{C}^{\text {low }} \sim 2\left\langle T_{C}^{\text {hom }}\right\rangle\right)$ with a 
much smaller weight. When increasing $x_{n s}$ to 0.04 , the width of the distribution is almost unaffected, but we notice a clear transfer of weight from the high $\mathrm{T}_{C}$ values to the lower one. By further increasing $x_{n s}$ to 0.06 , the transfer of weight increases further: the low- $\mathrm{T}_{C}$ region has a significantly higher weight. Finally for relatively high $x_{n s}(\sim 0.08)$, the weight is now concentrated around the lower $\mathrm{T}_{C}$ values and the distribution exhibits a tail-like structure at higher critical temperatures. This transfer of weight is the reason for the maximum in the $\mathrm{T}_{C}$ observed in Fig. 3(a), The origin of this kind of distribution is not very clear at first. However the analysis of the configurations reveals an interesting feature. We have considered two different kind of configurations. The first set of configurations of nanospheres corresponds to the situation where the distance between the nanospheres is restricted to small separations. The second kind corresponds to large separations between the nanospheres. First, it is found that in both cases the distribution of $\mathrm{T}_{C}$ is relatively narrow and unimodal. However, in the first case the $\mathrm{T}_{C}$ distribution is centered around $\mathrm{T}_{C}^{\text {low }}$, whereas in the second case it is centered around $\mathrm{T}_{C}^{h i g h}$. It is surprising and counter-intuitive that the largest $\mathrm{T}_{C}$ 's are obtained from the configurations where the inter nanosphere couplings are weaker. This is a clear indication that several length scales are competing. Now the nature of distributions shown in Fig. 4 can be explained as follows. In the case of low concentration of nanospheres (Fig. 4(a)) the probability of finding the nanospheres relatively far apart from each other is relatively high, and conversely the probability of finding them close to each other is relatively small. Thus this leads, in the distribution, to a significant weight around the high $\mathrm{T}_{C}$ values. As we gradually increase $x_{n s}$, the probability of finding configurations with the nanospheres at relatively large separation decreases, while the probability corresponding to small separation increases. As a consequence the weight in the distribution around $\mathrm{T}_{C}^{h i g h}$ decreases and that corresponding to the low $\mathrm{T}_{C}$ increases, as observed in Figs. 4(b) and 4(c). Finally for the largest $x_{n s}(\sim 0.08)$ the weight is mainly concentrated around the low- $\mathrm{T}_{C}$ region (Fig. 4(d)). Interestingly, this kind of behavior is not observed in the case of $\lambda=a$, for nanospheres of radii varying from $\mathrm{r}_{0}=a$ to $\sqrt{6} a$. For this case (longer range) the distribution of the critical temperatures is always narrow and unimodal, thus all the configurations (nanospheres far apart or close to each other) lead to similar values of the critical temperature. This confirms the idea that several length scales and typical couplings compete to give rise to this rich and new physics.

Let us now discuss some experimental consequences. We have shown that in systems 
with effective short-ranged exchange interactions it is possible to obtain two different critical temperatures depending on the size and concentration of the inhomogeneities and also on the typical separation between them. For example, in the case of $\lambda=a / 2$ for nanospheres of radii $\mathrm{r}_{0}=2 a, x_{n s}=0.02$ and $x_{i n}=0.8$, the $\mathrm{T}_{C}^{\text {high }}$ value is almost five times that of the $\mathrm{T}_{C}^{\text {low }}$ value. This could explain the wide range of $\mathrm{T}_{C}$ values observed experimentally for materials such as $(\mathrm{Ga}, \mathrm{Mn}) \mathrm{N}^{7-11}$ and the apparent dissension between theoretical predictions and experimental observations for these kinds of materials. In this context, it should be noted that Li et al. $\stackrel{29}{ }$ proposed two different ordering temperatures in $\mathrm{Ge}_{1-x} \mathrm{Mn}_{x}, \mathrm{~T}_{C}$ and $\mathrm{T}_{C}^{*}$ with $\mathrm{T}_{C} \ll \mathrm{T}_{C}^{*}$. The higher critical temperature $\mathrm{T}_{C}^{*}$ is associated with the ferromagnetic ordering temperature within isolated spin clusters and the onset of global ferromagnetism only occurs at $\mathrm{T}_{C}$. For $x=0.05$ the values of $\mathrm{T}_{C}$ and $\mathrm{T}_{C}^{*}$ were found to be 12 and $112 \mathrm{~K}$, respectively. However, detailed experimental studies in this direction would help to fully confirm this picture.

\section{CONCLUSION}

In conclusion, we have presented a detailed study of the effect of nanoscale inhomogeneities on the critical temperatures in diluted magnetic systems. We have shown that for materials with effective short ranged exchange interactions it is indeed possible to go beyond room temperature ferromagnetism by inducting nanoscale clusters of magnetic im-

purities. A gigantic increase in the critical temperatures of up to $1600 \%$, compared to that of the homogeneously diluted case, is obtained in certain cases. We also provide a plausible explanation for the wide variation of the $T_{C}$ 's, observed experimentally, in some materials such as $(\mathrm{Ga}, \mathrm{Mn})$ N. A meticulous study revealed that the relative separation between the inhomogeneities can play a decisive role in controlling the Curie temperatures. In some cases uniform distribution of nanospheres is found to favor very high critical temperatures. This fact could be further corroborated by detailed experimental studies. If, by controlling the growth conditions, the formation of the nanoscale inhomogeneities can be manipulated, it will open up the possibility of studying these disordered inhomogeneous systems in more detail. We believe that our study will pave the way for a better understanding of the origin and control of high-temperature ferromagnetism in dilute magnetic systems, which can serve as building blocks for potential future spintronic devices. 


\section{Acknowledgments}

We acknowledge Denis Feinberg, Claudine Lacroix, Arnaud Ralko and Paul Wenk for valuable discussions and insightful comments. S.K. gratefully acknowledges support by the WCU program (R31-2008-000-10059-0) AMS. A.C. would like to thank the Nanosciences Fondation for financial support.

* Electronic address: georges.bouzerar@grenoble.cnrs.fr

1 K. Sato, L. Bergqvist, J. Kudrnovský, P. H. Dederichs, O. Eriksson, I.Turek, B. Sanyal, G. Bouzerar, H. Katayama-Yoshida, V. A. Dinh, T. Fukushima,H. Kizaki and R. Zeller, Rev. Mod. Phys. 82, 1633 (2010).

2 T. Jungwirth, J. Sinova, J. Masek, J. Kucera and A. H. MacDonald, Rev. Mod. Phys. 78, 809 (2006).

3 C. Timm, J. Phys. Condens. Matter 15, R1865 (2003); C. Timm, F. Schäfer and F. von Oppen, Phys. Rev. Lett. 89, 137201 (2002).

4 K. Sato and H. Katayama-Yoshida, Semicond. Sci. Technol 17, 367 (2002).

5 T. Fukumura, H. Toyosaki and Y. Yamada, Semicond. Sci. Technol 20, S103 (2005).

6 S. A. Chambers, T. C. Droubay, C. M. Wang, K. M. Rosso, S. M. Heald, D. A. Schwartz, K. R. Kittilstved and D. R. Gamelin, Mater. Today 9, 28 (2006).

7 M.E. Overberg, C. R. Abernathy, S. J. Pearton, N. A. Theodoropoulou, K. T. McCarthy and A. F. Hebard, Appl. Phys. Lett. 79, 1312 (2001).

8 N. Theodoropoulou, A. F. Hebard, M. E. Overberg, C. R. Abernathy, S. J. Pearton, S. N. G. Chu, and R. G. Wilson, Appl. Phys. Lett. 78, 3475 (2001).

9 M. L. Reed, N. A. El-Masry, H. H. Stadelmaier, M. K. Ritums, M. J. Reed, C. A. Parker, J. C. Roberts and S. M. Bedair, Appl. Phys. Lett. 79, 3473 (2001).

10 G. T. Thaler, M. E. Overberg, B. Gila, R. Frazier, C. R. Abernathy, S. J. Pearton, J. S. Lee, S. Y. Lee, Y. D. Park, Z. G. Khim, J. Kim and F. Ren, Appl. Phys. Lett. 80, 3964 (2002).

11 S. Sonoda, S. Shimizu, T. Sasaki, Y. Yamamoto and H. Hori, J. Cryst. Growth 237-239, 1358 (2002).

12 R. Bouzerar and G. Bouzerar, Europhys. Lett. 92, 47006 (2010). 
13 G. Bouzerar, T. Ziman and J. Kudrnovský, Europhys. Lett. 69, 812 (2005).

14 L. Bergqvist, O. Eriksson, J. Kudrnovský, V. Drchal, P. Korzhavyi and I. Turek, Phys. Rev. Lett. 93, 137202 (2004).

15 K. Sato, W. Schweika, P. H. Dederichs and H. Katayama-Yoshida, Phys. Rev. B 70, 201202(R) (2004).

16 Y.D. Park, A. T. Hanbicki, S. C. Erwin, C. S. Hellberg, J. M. Sullivan, J. E. Mattson, T. F. Ambrose, A. Wilson, G. Spanos and B. T. Jonker, Science 295, 651 (2002).

17 A. P. Li, J. Shen, J. R. Thompson and H. H. Weitering, Appl. Phys. Lett. 86, 152507 (2005).

18 S. Cho, S. Choi, S. C. Hong, Y. Kim, J. B. Ketterson, B. J. Kim, Y. C. Kim and J. H. Jung, Phys. Rev. B 66, 033303 (2002).

19 N. Pinto, L. Morresi, M. Ficcadenti, R. Murri, F. D'Orazio, F. Lucari, L. Boarino and G. Amato, Phys. Rev. B 72, 165203 (2005).

20 F. Tsui, L. He, L. Ma, A. Tkachuk, Y. S. Chu, K. Nakajima and T. Chikyow, Phys. Rev. Lett. 91, $177203(2003)$.

21 J. S. Kang, G. Kim, S. C. Wi, S. S. Lee, S. Choi, S. Cho, S. W. Han, K. H. Kim, H. J. Song, H. J. Shin, A. Sekiyama, S. Kasai, S. Suga and B. I. Min, Phys. Rev. Lett. 94, 147202 (2005). 22 M. Jamet, A. Barski, T. Devillers, V. Poydenot, R. Dujardin, P. Bayle-Guillemaud, J. Rothman, E. Bellet-Amalric, A. Marty, J. Cibert, R. Mattana and S. Tatarenko, Nat. Mater. 5, 653 (2006).

23 D. Bougeard, S.Ahlers, A. Trampert, N. Sircar and G. Abstreiter, Phys. Rev. Lett. 97, 237202 (2006).

24 N. Jedrecy, H. J. von Bardeleben and D. Demaille, Phys. Rev. B 80, 205204 (2009).

25 K. Sato, H. Katayama-Yoshida and P. H. Dederichs, Jpn. J. Appl. Phys. 44, L948 (2005).

26 B. K. Rao and P. Jena, Phys. Rev. Lett. 89, 185504 (2002).

27 T. Hynninen, H. Raebiger, J. von Boehm and A. Ayuela, Appl. Phys. Lett. 88, 122501 (2006).

28 A. Chakraborty and G. Bouzerar, Phys. Rev. B 81, 172406 (2010).

29 A.P. Li, J.F. Wendelken, J. Shen, L. C. Feldman, J. R. Thompson and H. H. Weitering, Phys. Rev. B 72, 195205 (2005). 